\title{
ON NONLINEAR PROGRAMMING AND MATRIX GAME EQUIVALENCE
}

\author{
VASILE PREDA ${ }^{1}$
}

(Received 15 February 1991; revised 7 July 1992)

\begin{abstract}
In the framework of Mond-Weir duality a new equivalence between nonlinear programming and a matrix game is given. Finally, certain conclusions about convex programming with nested maxima and matrix games are also included.
\end{abstract}

\section{Introduction}

In Dantzig [3], some equivalence between linear programming duality and a symmetric matrix game is given. Kemp and Kimura [5] gave an equivalence theorem where the matrix game is not necessarily a symmetric game. In this case the matrix game depends on primal and dual variables. Chandra, Craven and Mond [2] presented analogues of results from [3] for certain class of nonlinear programming problems. The case of convex optimisation with nested maxima is treated in Preda [8].

The purpose of this note is to give analogues of Theorem 17 due to Kemp and Kimura [5, page 29] for a certain class of nonlinear programming problems, where matrix games depend only on primal variables. These problems are finite dimensional and satisfy certain generalised convexity requirements. Nonlinear programming problems with linear constraints are studied in Section 2, while those with nonlinear constraints are treated in Section 3. Finally, certain results about convex optimisation with nested maxima and matrix games are included in Section 4. This equivalence is given in the frame-work of Mond-Weir duality $[1,7]$.

\footnotetext{
'Mathematics Faculty, University of Bucharest, 14, Academiei Street, Bucharest, România (C) Australian Mathematical Society, 1994, Serial-fee code 0334-2700/94
} 


\section{Linear constraints case}

Let us consider the linearly constrained nonlinear programming problem (P) together with its Mond-Weir dual (D), as follows:

\begin{tabular}{l|l} 
(P) $\quad$ & $\begin{array}{l}\min f(x) \\
\text { subject to: } \quad A(x) \geq b, \quad x \geq 0 ;\end{array}$ \\
(D) & $\begin{array}{ll}\max g(x, u)=f(x)-u^{\top}(A x-b) \\
\text { subject to: } \quad \nabla f(x)-A^{\top} u \geq 0\end{array}$ \\
& $x^{\top}\left[\nabla f(x)-A^{\top} u\right] \leq 0, \quad u \geq 0$,
\end{tabular}

where $x \in \mathbb{R}^{n}, b \in \mathbb{R}^{m}, u \in \mathbb{R}^{m}, A=\left(a_{i j}\right)$ is an $m \times n$ real matrix, the symbol T denotes the transpose, $f: \mathbb{R}^{n} \rightarrow \mathbb{R}$ is differentiable and $\nabla f(x)$ denotes the gradient (column) vector of $f$ at $x$.

Now we consider the matrix game associated with the following $(n+1) \times$ $(m+1)$ matrix $M_{1}(x)$ (depending on $\left.x\right)$,

$$
M_{1}(x)=\left(\begin{array}{cc}
A^{\top} & \nabla f(x) \\
-b^{\top} & x^{\top} \nabla f(x)
\end{array}\right) .
$$

THEOREM 1. Let $P^{o}=\left(\begin{array}{c}x_{1}^{0} \\ z_{1}^{o}\end{array}\right), Q^{o}=\left(\begin{array}{c}u^{0} \\ z_{2}^{o}\end{array}\right), \bar{x}=x^{o} / z_{1}^{o}, \bar{u}=u^{o} / z_{2}^{o}$, with $z_{1}^{0}>0$, $z_{2}^{0}>0$. Let $\left(P^{o}, Q^{o}\right)$ solve the matrix game $M_{1}(\bar{x})$ and $P^{o \top} M_{1}(\bar{x}) Q^{o}=0$. Then $\bar{x}$ and $(\bar{x}, \bar{u})$ are feasible solutions to $(\mathrm{P})$ and $(\mathrm{D})$ respectively with $f(\bar{x})=$ $g(\bar{x}, \bar{u})$. In addition, if there is weak duality between (P) and (D) then $\bar{x}$ and $(\bar{x}, \bar{u})$ are optimal to the respective problems.

Proof. Because the value of the game (in random extension) is zero and $\left(P^{o}, Q^{o}\right)$ is an equilibrium point it results:

$$
M_{1}(\bar{x}) Q^{o} \leq 0 \quad \text { and } \quad M_{1}(\bar{x})^{\top} P^{o} \geq 0 .
$$

Hence

$$
\begin{aligned}
& A^{\top} u^{o}-z_{1}^{o} \nabla f(\bar{x}) \leq 0, \\
&-b^{\top} u^{o}+z_{1}^{o} \bar{x}^{\mathrm{T}} \nabla f(\bar{x}) \leq 0, \\
& A x^{o} \geq z_{2}^{o} b, \\
&-x^{o \top} \nabla f(\bar{x})+z_{2}^{o} \bar{x}^{\mathrm{T}} \nabla f(\bar{x}) \geq 0 .
\end{aligned}
$$


But $x^{o} \geq 0, u^{o} \geq 0, z_{1}^{o}>0, z_{2}^{o}>0$ and therefore from above we obtain

$$
\begin{aligned}
A^{\top} \bar{u}-\nabla f(\bar{x}) & \leq 0, \\
-b^{\top} \bar{u}+\bar{x}^{\top} \nabla f(\bar{x}) & \leq 0, \\
A \bar{x} & \geq b, \\
-\bar{x}^{\top} \nabla f(\bar{x})+\bar{x}^{\top} \nabla f(\bar{x}) & \geq 0 .
\end{aligned}
$$

We further observe from the above

$$
\begin{gathered}
\bar{x}^{\top} \nabla f(\bar{x}) \leq b^{\top} \bar{u} \leq \bar{x}^{\top} A^{\top} \bar{u} \leq \bar{x}^{\top} \nabla f(\bar{x}), \quad \text { i.e., } \\
b^{\top} \bar{u}=\bar{x}^{\top} A^{\top} \bar{u}=\bar{x}^{\top} \nabla f(\bar{x}) .
\end{gathered}
$$

Now, $g(\bar{x}, \bar{u})=f(\bar{x})-\bar{u}^{\top}(A \bar{x}-b)=f(\bar{x})$.

By using (5)-(9) we have: $\bar{x}^{\mathrm{T}}\left[\nabla f(\bar{x})-A^{\mathrm{\top}} \bar{u}\right]=0$. Thus $\bar{x},(\bar{x}, \bar{u})$ are feasible solutions for $(\mathrm{P})$ and $(\mathrm{D})$ respectively. When a weak duality exists between $(\mathrm{P})$ and (D) then $\bar{x}$ is optimal for (P) and $(\bar{x}, \bar{u})$ is optimal for (D).

THEOREM 2. Let $\bar{x}$ and $(\bar{x}, \bar{u})$ be feasible solutions to $(\mathrm{P})$ and $(\mathrm{D})$ respectively, such that $\bar{u}^{\top}(A \bar{x}-b)=0$. We define

$$
\begin{array}{cc}
z_{1}^{o}=1 /\left(1+\sum_{i=1}^{n} \bar{x}_{1}\right), & z_{2}^{o}=1 /\left(1+\sum_{j=1}^{m} \bar{u}_{j}\right), \\
P^{o}=\left(\begin{array}{c}
\bar{x} z_{1}^{o} \\
z_{1}^{o},
\end{array}\right), & Q^{o}=\left(\begin{array}{c}
\bar{u} z_{2}^{o} \\
z_{2}^{o}
\end{array}\right) .
\end{array}
$$

Then $\left(P^{o}, Q^{o}\right)$ solve the matrix game $M_{1}(\bar{x})$ and the value of this game is zero.

PROOF. We have

$$
\begin{aligned}
P^{o \top} M_{1}(\bar{x}) Q^{o} & =\left(z_{1}^{o} \bar{x}^{\top} A^{\top}-z_{1}^{o} b^{\top},-z_{1}^{o} \bar{x}^{\top} \nabla f(\bar{x})+z_{1}^{o} \bar{x}^{\top} \nabla f(\bar{x})\right)\left(\begin{array}{c}
\bar{u} z_{2}^{o} \\
z_{2}^{o}
\end{array}\right) \\
& =z_{1}^{o} z_{2}^{o}\left[\bar{x}^{\top} A^{\top} \bar{u}-b^{\top} \bar{u}-\bar{x}^{\top} \nabla f(\bar{x})+\bar{x}^{\top} \nabla f(\bar{x})\right] \\
& =z_{1}^{o} \cdot z_{2}^{o} \cdot \bar{u}^{\top}(A \bar{x}-b)=0 .
\end{aligned}
$$

Because $\bar{x}$ and $(\bar{x}, \bar{u})$ are feasible solutions to (P) and (D) respectively, and $\bar{u}^{\top}(A \bar{x}-b)=0$, we obtain

$$
\bar{x}^{\top} A^{\top} \bar{u}=\bar{x}^{\top} \nabla f(\bar{x})=b^{\top} \bar{u} .
$$


Now (10) and the conditions of the theorem relative to $\bar{x},(\bar{x}, \bar{u})$ give

$$
\begin{aligned}
& M_{1}(\bar{x}) Q^{o}=z_{2}^{o}\left(\begin{array}{c}
A^{\top} \bar{u}-\nabla f(\bar{x}) \\
-b^{\top} \bar{u}+\bar{x}^{\top} \nabla f(\bar{x})
\end{array}\right) \leq 0, \\
& P^{o \top} M_{1}(\bar{x})=z_{1}^{o} \cdot\left(\bar{x}^{\top} A^{\top}-b,-\bar{x}^{\top} \nabla f(\bar{x})+\bar{x}^{\top} \nabla f(\bar{x})\right) \geq 0 .
\end{aligned}
$$

Thus $\left(P^{o}, Q^{o}\right)$ is an equilibrium point for the matrix game $M_{1}(\bar{x})$ and the value of this game is zero.

\section{Nonlinear constraints case}

Let us consider the general nonlinear programming problem (NP) together with its dual (ND) as follows

$$
\begin{array}{l|l}
\text { (NP) } & \begin{array}{l}
\min f(x) \\
\text { subject to: } \quad a(x) \leq 0, \quad x \geq 0 ;
\end{array}
\end{array} \quad \text { (ND) } \quad \begin{array}{ll}
\max g(x, u)=f(x)+u^{\top} a(x) \\
\text { subject to: } \quad \nabla f(x)+\nabla a(x) u \geq 0 \\
& x^{\top}[\nabla f(x)+\nabla a(x) u] \leq 0, \quad u \geq 0,
\end{array}
$$

where $x, u, f$ are the same as in Section $2, a: \mathbb{R}^{n} \rightarrow \mathbb{R}^{m}$ is a convex and continuously differentiable function, and the gradient $\nabla a(x)$ is an $n \times m$ matrix.

Define now the $(n+1) \times(m+1)$ matrix $M_{2}(x)$ by

$$
M_{2}(x)=\left(\begin{array}{cc}
-\nabla a(x) & -\nabla f(x) \\
x^{\top} \nabla a(x)-(a(x))^{\top} & x^{\top} \nabla f(x)
\end{array}\right) .
$$

Now we have

THEOREM 3. Let $P^{o}=\left(\begin{array}{c}x^{o} \\ z_{1}^{o}\end{array}\right), Q^{o}=\left(\begin{array}{c}u^{o} \\ z_{2}^{o}\end{array}\right), \bar{x}=x^{o} / z_{1}^{o}, \bar{u}=u^{o} / z_{2}^{o}$ with $z_{1}^{o}>0$, $z_{2}^{o}>0$. Let $\left(P^{o}, Q^{o}\right)$ be an equilibrium point for the matrix game $M_{2}(\bar{x})$ and $P^{o \mathrm{~T}} M_{2}(\bar{x}) Q^{o}=0$. Then $\bar{x}$ and $(\bar{x}, \bar{u})$ are feasible solutions to (NP) and (ND) respectively, with the two objective functions having equal values. If also weak duality holds between (NP) and (ND), then $\bar{x}$ is optimal for (NP) and $(\bar{x}, \bar{u})$ is optimal for (ND).

Proof. Because $\left(P^{o}, Q^{o}\right)$ is an equilibrium point for $M_{2}(\bar{x})$ and $P^{o \top} M_{2}(\bar{x}) Q^{o}=$ 0 we obtain: $M_{2}(\bar{x}) Q^{o} \leq 0$ and $P^{o \top} M_{2}(\bar{x}) \geq 0$, i.e.,

$$
-z_{2}^{o} \nabla a(\bar{x}) \bar{u}-z_{2}^{o} \nabla f(\bar{x}) \leq 0,
$$




$$
\begin{aligned}
z_{2}^{o} \bar{x}^{\top} \nabla a(\bar{x}) \bar{u}-z_{2}^{o} a(\bar{x})^{\top} \bar{u}+z_{2}^{o} \bar{x}^{\top} \nabla f(\bar{x}) & \leq 0, \\
-z_{1}^{o} \cdot \bar{x}^{\top} \nabla a(\bar{x})+z_{1}^{o} \cdot \bar{x}^{\top} \nabla a(\bar{x})-z_{1}^{o} a(\bar{x})^{\top} \geq 0, & \geq z_{1}^{o} \cdot \bar{x}^{\top} \nabla f(\bar{x})+z_{1}^{o} \cdot \bar{x}^{\top} \nabla f(\bar{x}) \geq 0 .
\end{aligned}
$$

Because $z_{1}^{o}>0$ by (13) we obtain $a(\bar{x}) \leq 0$. But $\bar{x} \geq 0$. Hence $\bar{x}$ is a feasible solution for (NP). By $\bar{x} \geq 0$ and (11) we get $-z_{2}^{o} \bar{x}^{\top} \nabla a(\bar{x}) \bar{u}-z_{2}^{o} \bar{x}^{\top} \nabla f(\bar{x}) \leq 0$ and then (12) gives $\bar{u}^{\top} a(\bar{x}) z_{2}^{o} \geq 0$. Dividing this relation by $z_{2}^{o}>0$ we obtain $\bar{u}^{\mathrm{T}} a(\bar{x}) \geq 0$. Using this relation together with $\bar{u} \geq 0$ and $a(\bar{x}) \leq 0$ we obtain

$$
\bar{u}^{\top} a(\bar{x})=0 .
$$

From (15) and dividing (11) and (12) by $z_{2}^{o}>0$ we obtain $(\bar{x}, \bar{u})$ is feasible for (ND). Moreover, according to (15), we have $g(\bar{x}, \bar{u})=f(\bar{x})+\bar{u}^{\mathrm{T}} a(\bar{x})=f(\bar{x})$, i.e., the two objective functions have equal values. This, with weak duality, proves that $\bar{x}$ is optimal for (NP) and $(\bar{x}, \bar{u})$ is optimal for (ND).

THEOREM 4. Let $\bar{x}$ and $(\bar{x}, \bar{u})$ be feasible solutions to (NP) and (ND) respectively, with $\bar{u}^{\mathrm{T}} a(\bar{x})=0$. Let

$$
\begin{array}{cc}
z_{1}^{o}=1 /\left(1+\sum_{i=1}^{n} \bar{x}_{i}\right), & z_{2}^{o}=1 /\left(1+\sum_{j=1}^{m} \bar{u}_{J}\right), \\
P^{o}=\left(\begin{array}{c}
z_{1}^{o} \bar{x} \\
z_{1}^{o}
\end{array}\right), & Q^{o}=\left(\begin{array}{c}
z_{2}^{o} \bar{u} \\
z_{2}^{o}
\end{array}\right) .
\end{array}
$$

Then $\left(P^{o}, Q^{o}\right)$ solve the matrix game $M_{2}(\bar{x})$ and the value of this game is zero.

PROOF. From the hypotheses of the theorem, we have

$$
\begin{aligned}
& P^{o \top} M_{2}(\bar{x}) Q^{o}=\left(-z_{1}^{o} \bar{x}^{\top} \nabla a(\bar{x})-z_{1}^{o} a(\bar{x})^{\top}+z_{1}^{o} \bar{x}^{\top} \nabla a(\bar{x}),-z_{1}^{o} \bar{x}^{\top} \nabla f(\bar{x})\right. \\
& \left.+z_{1}^{o} \bar{x}^{\top} \nabla f(\bar{x})\right) Q^{o} \\
& =z_{1}^{o} \cdot z_{2}^{o}\left[-\bar{x}^{\mathrm{T}} \nabla a(\bar{x}) \bar{u}+\bar{x}^{\mathrm{\top}} \nabla a(\bar{x}) \bar{u}-a(\bar{x})^{\mathrm{\top}} \bar{u}\right. \\
& \left.-\bar{x}^{\top} \nabla f(\bar{x})+\bar{x}^{\top} \nabla f(\bar{x})\right]=0, \\
& M_{2}(\bar{x}) Q^{o}=-z_{2}^{o} \cdot\left(\begin{array}{c}
-\nabla a(\bar{x}) \bar{u}+\nabla f(\bar{x}) \\
a(\bar{x})^{\top} \bar{u}
\end{array}\right) \leq 0, \\
& P^{o \top} M_{2}(\bar{x})=-z_{1}^{o}\left(\bar{x}^{\top} \nabla a(\bar{x})-\bar{x}^{\top} \nabla a(\bar{x})+a(\bar{x})^{\top}, \bar{x}^{\top}[\nabla f(\bar{x})-\nabla f(\bar{x})]\right) \\
& =-z_{1}^{o}\left(a(\bar{x})^{\top}, 0\right) \geq 0 .
\end{aligned}
$$

Thus, $\left(P^{o}, Q^{o}\right)$ is an equilibrium point for $M_{2}(\bar{x})$ and the value of this game is zero. 
REMARK 1. The hypothesis of Theorems 1 and 3 seems to require a knowledge of $\bar{x}$ in order to find $\bar{x}$, but such results are not uncommon in nonlinear programming (see [4], [6], [2, page 126]).

REMARK 2. The hypothesis of Theorems 1 and 3 assure that the Kuhn-Tucker conditions in $\bar{x}$ are verified and $(\bar{x}, \bar{u})$ is feasible solution for the Wolfe dual of the problems (P) and (NP) respectively.

\section{Convex constraints with the nested maxima case}

Let us consider the following programming problem

$$
\mid \begin{aligned}
& \min f(x)=\Psi(x)+\max _{1 \leq i \leq k} c_{i}(x) \\
& \text { subject to: } \quad a(x) \leq 0, \quad x \geq 0 ;
\end{aligned}
$$

where $\Psi: \mathbb{R}^{n} \rightarrow \mathbb{R}, c_{l}: \mathbb{R}^{n} \rightarrow \mathbb{R}, 1 \leq i \leq k, a: \mathbb{R}^{n} \rightarrow \mathbb{R}^{m}$, are convex and continuously differentiable functions.

This problem is equivalent with

$$
\mid \begin{array}{lll}
\min g(x, y)= & \Psi(x)+y & \\
\text { subject to: } & a(x) \leq 0, & c(x)-e y \leq 0, \\
& x \geq 0, & y \in \mathbb{R},
\end{array}
$$

where $c(x)=\left(c_{1}(x), \ldots, c_{k}(x)\right)^{\top}$ and $e=(1, \ldots, 1)^{\top} \in \mathbb{R}^{k}$.

For (P2), the Mond-Weir dual is defined by

(D1)

$$
\begin{array}{|l}
\max \left[g(x, y)+u^{\top} a(x)+v^{\top}(c(x)-e y)\right] \\
\text { subject to: } \quad \nabla \Psi(x)+\nabla a(x)+\nabla c(x) v \geq 0 \\
\\
\quad e^{\top} v=1 \\
x^{\top}[\nabla \Psi(x)+\nabla a(x) u+\nabla c(x) v]+y\left(1-e^{\top} v\right) \leq 0 \\
u \geq 0, v \leq 0, x \in \mathbb{R}, y \in \mathbb{R} .
\end{array}
$$

Here, $\nabla c(x)$ is a $n \times m$ matrix.

On the other hand, to obtain in this case a matrix game, we consider the following problem equivalent with (P2):

$$
\begin{aligned}
& \min h\left(x, y_{1}, y_{2}\right)=\Psi(x)+y_{1}-y_{2} \\
& \text { with the constraints: } \quad a(x) \leq 0 \text {, } \\
& c(x)-e y_{1}+e y_{2} \leq 0 \text {, } \\
& x \geq 0, \quad y_{1} \geq 0, \quad y_{2} \geq 0 \text {. }
\end{aligned}
$$


Now for this case we define the matrix game $M_{3}(x, y)$,

$$
M_{3}(x, y)=\left(\begin{array}{ccc}
-\nabla a(x) & -\nabla c(x) & -\nabla \Psi(x) \\
0 & e^{\top} & -1 \\
0 & -e^{\top} & 1 \\
x^{\top} \nabla a(x)-a(x)^{\top} & x^{\top} \nabla c(x)-c(x)^{\top} & -x^{\top} \nabla \Psi(x)+y
\end{array}\right) .
$$

By using the procedure of Section 3, it can be verified that the relations between primal-dual pair (P1), (D1) and the matrix game $M_{3}(x, y)$ given by Theorems 5 and 6 below hold.

THEOREM 5. Let $P^{o \top}=\left(x^{o \top}, y_{1}^{o \top}, y_{2}^{o \top}, z_{1}^{o}\right), Q^{o \top}=\left(u^{o \top}, v^{o \top}, z_{2}^{o}\right), \bar{x}=x^{o} / z_{1}^{o}$, $\bar{y}=\left(y_{1}^{o}-y_{2}^{o}\right) / z_{1}^{o}, \bar{u}=u^{o} / z_{2}^{o}, \bar{v}=v^{o} / z_{2}^{o}$. Let $\left(P^{o}, Q^{o}\right)$ be an equilibrium point for the matrix game $M_{3}(\bar{x}, \bar{y})$ and the value of this game be zero. Then

a) $(\bar{x}, \bar{y})$ and $(\bar{x}, \bar{y}, \bar{u}, \bar{v})$ are feasible solutions to (P2) and (D1) respectively with $f(\bar{x})=g(\bar{x}, \bar{y})+\bar{u}^{\top} a(\bar{x})+\bar{v}^{\top}(c(\bar{x})-e \bar{y})$;

b) $\bar{x}$ is a feasible solution to (P1);

c) if weak duality holds between (P1) and (D1), then $\bar{x}$ and $(\bar{x}, \bar{y}, \bar{u}, \bar{v})$ are optimal for $(\mathrm{P} 1)$ and $(\mathrm{D} 1)$ respectively.

THEOREM 6. Let $\bar{x}$ be a feasible solution to (P1) and $\bar{y} \in \mathbb{R}$ such that $(\bar{x}, \bar{y})$ and $(\bar{x}, \bar{y}, \bar{u}, \bar{v})$ are feasible solutions to $(\mathrm{P} 2)$ and $(\mathrm{D} 1)$ respectively. We suppose that: $\bar{u}^{\top} a(\bar{x})=0, \bar{v}^{\top}(c(\bar{x})-e \bar{y})=0$. Let us define: $z_{1}^{o}=1 /\left(1+\sum_{i=1}^{n} \bar{x}_{i}+\bar{y}\right)$, $z_{2}^{o}=1 /\left(1+\sum_{j=1}^{m} \bar{u}_{j}+\sum_{s=1}^{k} \bar{v}_{s}\right)$. Then there exist $\bar{y}_{1} \geq 0, \bar{y}_{2} \geq 0$ with $\bar{y}=\bar{y}_{1}-\bar{y}_{2}$, such that $\left(P^{o}, Q^{o}\right)$ solve the matrix game $M_{3}(\bar{x}, \bar{y})$ and the value of this game is zero, where $P^{o \top}=z_{1}^{o} \cdot\left(\bar{x}^{\top}, \bar{y}_{1}^{\top}, \bar{y}_{2}^{\top}, 1\right), Q^{o \top}=z_{2}^{o}\left(\bar{u}^{\top}, \bar{v}^{\top}, 1\right)$.

REMARK 3. There is a weak duality between (P2) and (D1) because $g(x, y)+$ $u^{\top} a(x)+v^{\top}(c(x)-e y)$ is a convex function of $x, y$, for all feasible $x, y, u, v$.

REMARK 4. If $\Psi \equiv 0$, we obtain a minmax problem.

REMARK 5. For fractional programs similar comments and conclusions hold.

\section{Some further remarks and results}

In this section we consider some remarks relative to conditions $P^{o \top} M_{1}(\bar{x}) Q^{o}$ $=0$ (Theorem 1), $\bar{u}^{\top}(A \bar{x}-b)=0$ (Theorem 2) and the way to obtain an information about compatibility of inequality systems. 
REMARK 6. Relative to Theorem 1 , if $m=n, A^{\top}=-A, \bar{x}$ is a stationary point for $f$ and $b \geq 0$, then the value of matrix game $M_{1}(\bar{x})$ is zero. Hence the condition $P^{o \top} M_{1}(\bar{x}) Q^{o}=0$ is satisfied.

REMARK 7. Relative to Theorem 2, if $(\bar{x}, \bar{u})$ is a feasible solution for (D) and a saddle point for the Lagrangian of problem $(\mathrm{P})$, then the condition $\bar{u}^{\mathrm{T}}(A \bar{x}-b)=$ 0 is satisfied.

REMARK 8. By virtue of theorems above, useful ideas from both theory and mathematical programming can be applied to problems in either discipline. Now we consider an application of the matrix game $M_{1}(x)$ to the inequality systems.

Let us first consider a general lemma. In the following we use some notations. If $x=\left(x_{1}, \ldots, x_{n}\right)^{\top}$ is a vector, then $x \geq 0$ shall mean $x_{t} \geq 0$ for all $i=1,2, \ldots, n$; $x>0$ shall signify $x_{i} \geq 0$ and $x \neq 0 ; x \gg 0$ denotes that $x_{i}>0$ for all $i=1,2, . ., n$.

LEMMA 1. Let $C$ be a matrix game $s \times p$. Then, between the following situations (i1), (i2) and (i3), one and only one is true:

(i1) the system $c \eta \ll 0, \eta>0, \eta \in \mathbb{R}^{p}$, is compatible;

(i2) the systems

$$
\text { (a) } \mid \begin{aligned}
& C \eta \leq 0, \\
& \eta>0, \\
& \eta \in \mathbb{R}^{p}
\end{aligned} \quad \text { and (b) } \mid \begin{aligned}
& C^{\top} \xi \geq 0 \\
& \xi>0, \\
& \xi \in \mathbb{R}^{s}
\end{aligned}
$$

are compatible;

(i3) the system $C^{\top} \xi \gg 0, \xi>0, \xi \in \mathbb{R}^{S}$, is compatible.

PROOF. According to the minmax theorem for matrix games we have that there exists an equilibrium point $(\xi, \eta)$, in the mixed strategies, and a real number $v$ such that

$$
C \eta \leq v e_{s} \text { and } v e_{p} \leq C^{\top} \xi,
$$

where $e_{s}=(1,1, \ldots, 1)^{\top} \in \mathbb{R}^{s}, e_{p}=(1, \ldots, 1)^{\top} \in \mathbb{R}^{p}$.

By (16) we obtain (i1), (i2) and (i3) according as $v<0,=0$ and $>0$.

Hence, between statements (i1), (i2) and (i3), at least one is true. If (i1) and (i2) are both true then by (i1) and (b) we obtain $\xi^{\top} C \eta \geq 0$. But $C \eta \ll 0$ and $\xi \geq 0, \xi \neq 0$. Thus $\xi^{\top} C \eta<0$. Hence we have a contradiction. 
In the same way we obtain that (i1) and (i3), (i2) and (i3) respectively, are not both true.

Using this lemma and $M_{3}(x)$, it is possible to consider a result relative to compatibility of some inequality systems. Here we consider a special case for $M_{2}(x)$.

PROPOSITION 1. Let us consider $A$, an $m \times n$ real matrix and $b \in \mathbb{R}^{m}$. Then, between the systems

$$
\begin{array}{l|l}
\text { and } \quad \begin{array}{l}
A \alpha_{1}=\alpha_{2} b \\
\alpha_{1}, \alpha_{2} \geq 0 \\
\left(\alpha_{1}, \alpha_{2}\right) \neq 0 \\
\alpha_{1} \in \mathbb{R}^{n}, \quad \alpha_{2} \in \mathbb{R}
\end{array} \\
\begin{array}{l}
A^{\top} \gamma \ll 0 \\
b^{\top} \gamma>0 \\
\gamma \in \mathbb{R}^{m},
\end{array}
\end{array}
$$

one and only one is compatible.

PROOF. Let $\mathrm{f}$ be a constant function on $\mathbb{R}^{n}$ and the problem: $\inf (f(x) / A x=$ $b, x \geq 0$ ). This problem is equivalent to $\inf (f(x) / A x \geq b,-A x \geq-b, x \geq 0$ ). Then for any $x$, the matrix $M_{1}(x)$ is defined by

$$
M_{1}(x)=\left(\begin{array}{ccc}
A^{\top} & -A^{\top} & 0 \\
-b^{\top} & b^{\top} & 0
\end{array}\right)
$$

According to Lemma 1 at least one of the following statements is true:

(j1) the system

$$
\mid \begin{aligned}
& A^{\top} \gamma \ll 0 \\
& b^{\top} \gamma>0 \\
& \gamma \in \mathbb{R}^{m}
\end{aligned}
$$

is compatible, and

(j2) the systems

$$
\mid \begin{array}{l|l}
A^{\top} \gamma \leq 0 \\
b^{\top} \gamma \geq 0 \\
\gamma \in \mathbb{R}^{m}
\end{array} \quad \text { and } \quad \mid \begin{aligned}
& A \alpha_{1}=\alpha_{2} b \\
& \alpha_{1}, \alpha_{2} \geq 0 \\
& \left(\alpha_{1}, \alpha_{2}\right) \neq 0 \\
& \alpha_{1} \in \mathbb{R}^{n}, \quad \alpha_{2} \in \mathbb{R}
\end{aligned}
$$

are compatible.

Now is sufficient to prove that the systems are not compatible simultaneously. If $\left(\alpha_{1}, \alpha_{2}\right)$ is a solution to (17) and $\gamma$ is a solution to (18) then $\alpha_{1}^{\top} A^{\top} \gamma=\alpha_{2} b^{\top} \gamma$. 
By this relation together with $\left(\alpha_{1}, \alpha_{2}\right)>0, b^{\top} \gamma>0$ and $A^{\top} \gamma \ll 0$ we obtain a contradiction.

REMARK 9. We note that (17) is compatible if and only if at least one of the following systems is compatible:

$$
\begin{aligned}
& A \alpha_{1}=b \\
& \alpha_{1} \geq 0 \\
& \alpha_{1} \in \mathbb{R}^{n} \\
& \mid \begin{aligned}
A \alpha_{1} & =0 \\
\alpha_{1} & >0 \\
\alpha_{1} & \in \mathbb{R}^{n} .
\end{aligned}
\end{aligned}
$$

\section{Acknowledgement}

The author is indebted to the referee and Prof. B. D. Craven for various corrections and some suggestions to revise the paper.

\section{References}

[1] S. Chandra, B. D. Craven and B. Mond, "Generalized concavity and duality with a square root term", Optimization 16 (1985) 653-662.

[2] S. Chandra, B. D. Craven and B. Mond, "Non-linear programming duality and matrix game equivalence", J. Austral. Math. Soc. Ser. B 26 (1985) 422-429.

[3] G. B. Dantzig, "A proof of the equivalence of the programming problem and the game problem", in Activity analysis of production and allocation (ed. T. C. Koopmans), Cowles Commission Monograph No. 13, (John Wiley and Sons, 1951).

[4] S. Karlin, Mathematical methods and theory in games, programming and mathematical economics (Addison-Wesley, Reading, Mass., 1959).

[5] M. Kemp and Y. Kimura, Introduction to mathematical economics (Springer Verlag, New York, 1978).

[6] K. O. Kortanek and J. P. Evans, "Pseudo-concave programming and convex Lagrange regularity", Oper. Res. 15 (1967) 882-891.

[7] B. Mond and T. Weir, "Generalized concavity and duality", in Generalized concavity in optimization and economics (eds. S. Schaible and W. T. Ziemba), (Academic Press, 1981), 263-279.

[8] V. Preda, "Convex optimization with nested maxima and matrix game equivalence", Annals of Bucharest University 3 (1989) 57-60.

[9] C. H. Scott, T. R. Jefferson and E. Sirri, "On duality for convex minimization with nested maxima", J. Austral. Math. Soc. Ser. B 26 (1985) 517-522. 